\title{
Modified Haar Wavelet based Method for Compressing Medical Images
}

\author{
P.Chitra $1^{\# 1}$, M.Mary Shanthi Rani $2^{\# 2, *}$ \\ \#1 Department of Computer Science and Applications, \\ The Gandhigram Rural Institute- Deemed to be University, \\ Tamil Nadu-624 302 \\ \#2 Department of Computer Science and Applications, \\ The Gandhigram Rural Institute- Deemed to be University, \\ Tamil Nadu-624 302
}

\section{ABSTRACT}

Nowadays, many researchers are attracted by image compression techniques because of the minimization of storage and transmission cost over the internet. The main objective of image compression is to reduce the redundant and irrelevant information from the original data. Haar Wavelet Transformation (HWT) is an efficient transform coding among the lossy image transform techniques. This proposed work has two stages of process: Vector Quantization and Thresholding. In the first stage, Haar Wavelet Transformation is implemented which results categorized into two matrices; Approximation Co-efficient (AC) matrix and Detailed Coefficient (DC) matrices. The second stage of proposed method is applied Vector Quantization(VQ) to improve the compression efficiency. This proposed work achieved higher compression ratio without compromising the image quality. The performance of the proposed method is compared using the traditional Haar Wavelet transform based method which has been evaluated using some popular parameters such as Peak Signal to Noise Ratio (PSNR), Structural Similarity Index(SSIM), Compression Ratio (CR) and Bit Rate(BR). The computation cost is minimized using proposed method. The comparative analysis of the proposed method has witnessed the achievement of good compression ratio and image quality as well.

Key words: Image Compression, Haar Wavelet Transform, Vector Quantization, Run Length Encoding (RLE), Thresholding Technique.

Corresponding Author: Dr. M.Mary Shanthi Rani, Asst. Professor, DCS\&A, GRI-DU

\section{INTRODUCTION}

Image compression creates a fine solution for the problem of massive data storage and transmission. Nowadays, the people need enormous data to send and receive through the internet. The main process of image compression is the reduction of redundant information from the original data [1]. It is also used to reduce the size of the original image data in bytes without much degradation in the quality of the image. 
DOI: https://dx.doi.org/10.26808/rs.ca.i8v1.25

The encoder creates a set of symbols representing the input image $f(x, y)$. The decoder at the receiving end uses the symbols to reconstruct the input image. The basic process of image compression is shown in Fig.1.

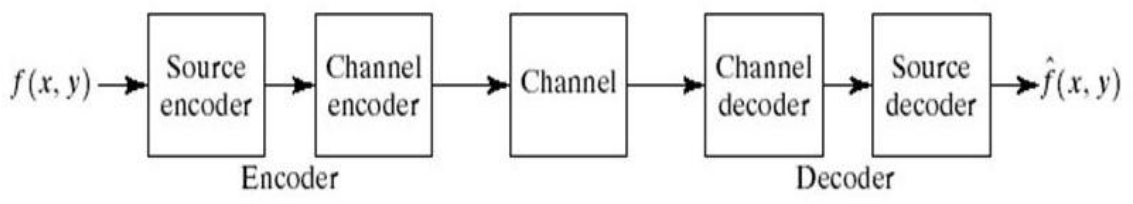

Fig 1. General process of Image Compression

Generally, the compression process can be classified into lossless or lossy [2]. In lossless compression, original image is perfectively reconstructed without loss any information. In lossy compression, there is some loss in the form of redundant and irrelevant information from the original image. Haar Wavelet Transform is one of the simple lossy compression technique. Several methods are proposed for compressing images based on vector quantization [3-5] and transforms[6].

\section{HAAR WAVELET TRANSFORM}

Haar Wavelet is a one of the traditional wavelet technique to extend the quality of an image. The process of haar wavelet is easy to manage the complex task through their performance. It has 2 different outputs like approximation (average) coefficient matrix and Detail (difference) coefficient matrices. General representation of the Haar wavelet is visually presented in Fig.2.

\begin{tabular}{|c|c|}
\hline $\begin{array}{c}\text { Approximation } \\
\text { Coefficient }\end{array}$ & $\begin{array}{c}\text { Detail } \\
\text { Coefficient } \\
\text { (Horizontal) }\end{array}$ \\
\hline $\begin{array}{c}\text { Detail } \\
\text { Coefficient } \\
\text { (Vertical) }\end{array}$ & $\begin{array}{c}\text { Detail } \\
\text { Coefficient } \\
\text { (Diagonal) }\end{array}$ \\
\hline
\end{tabular}

Fig 2. Decomposition of Haar Wavelet Transform

The principle behind this concept of Haar wavelet is easy to calculate the average and differences of neighbourhood pixels.

\section{VECTOR QUANTIZATION}

Vector quantization(VQ) is a lossy compression method. known for its ease of implementation and efficiency. It achieves compression by representing a large set of vectors with a few vectors called the codebook which is generated through the encoding process. The decoder, at the receiving end uses the code vectors and the code indices to reconstruct the input image. The performance of the VQ depends on the quality of the codebook which is influenced by several 
DOI: https://dx.doi.org/10.26808/rs.ca.i8v1.25 International Journal of Computer Application (2250-1797) Issue 8 Volume 1, January- February 2018

factors like initial seeds, number of iterations and vector dimension. The process of vector quantization is presented in Fig.3.

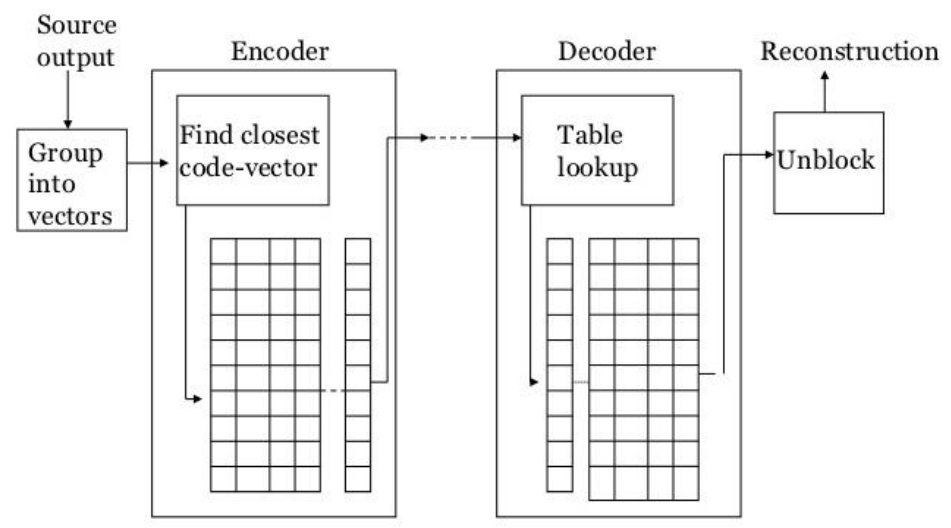

Fig 3. Vector Quantization process

\section{RELATED WORK}

There are various existing methods are proposed using Haar wavelet and RVQ techniques.

Gopal Lakhani.,[7] proposed a novel technique based on JPEG arithmetic coding (JAC) algorithm for enhancing image quality. L. Li., et al. [8] developed a prediction model based on shanon's rate-distortion theorem. Kharate., et al. [9] proposed an algorithm of wavelet packet entropy best tree based on threshold entropy with lossy enhanced run length encoding. Deng Wang., et al. [10] developed a novel image coding system in which Original EEG signals are represented by wavelet packet coefficients and feature extraction using the best basis-based wavelet packet entropy method. Mukesh Mittal., et al.[11] presented a review of various vector quantization algorithms used for the designing of the codebook to obtain better quality. D.Aruna Kumari., et al. [12] proposed a technique for preserving data and has described the process of quantization on training data samples that will produce transformed data set. S.Vimala., [13] developed a novel algorithm based on vector quantization technique to enhance the quality of image. The author describes about the high detail blocks which are identified based on the maximum and minimum pixel values of the blocks.

The rest of the paper is structured as follows: Section 3 describes the process of the proposed method in a detail form of description about the methodology. Performance and comparative analysis of the proposed method is presented in Section 4. The merits and future enhancements are pointed out in Section 5.

\section{PROPOSED WORK}

In this proposed work, investigates a novel methodology using Haar Wavelet Transform (HWT) and Thresholding. Generally, the Haar wavelet transformation has a set of four coefficient matrices like Approximation Co-efficient(AC) matrix and three Detail Co-efficient(DC) matrices. These matrices are further subjected to achieve compression to enhance compression ratio. The approximation co-efficient matrix is compressed using Vector Quantization(VQ) and DC Matrices are compressed using Run-length coding prior to compression, The DC matrices 
DOI: https://dx.doi.org/10.26808/rs.ca.i8v1.25

are converted to sparse matrices by applying thresholding and the sparse DC matrices are compressed using run length coding in the next stage.

\section{The Proposed Method (HWT_VQ)}

The proposed method work is briefly described in three stages.

Stage 1: Haar Wavelet Transformation of the input image

Stage 2:Compression of Approximation Coefficient matrix obtained in stage1 using Vector quantization

Stage 3:Compression of Detail Coefficient(DC) matrices obtained in stage1 using thresholding and run length encoding.

Stage One: The input images is read and transformed by applying HWT, The output of HWT has four matrices one AC matrix and three DC matrices

Stage Two: The AC matrix obtained from Stage 1 is compressed further by applying Vector Quantization. K-means clustering algorithm is used to generate the codebook, set of cluster centroids. The AC matrix is divided into small blocks of size (block size*block size), The value of block size may be chosen based on the desired image quality for good image quality. The block size should be kept small for achieving good quality image, The training vectors (blocks) are clustered using K-means algorithm The output of K-means is a set of optimal cluster centroids which form the codebook. The size of the codebook also determine the image quality.

Stage Three : The three DC matrices are subject to hard thresholding to make the DC matrices more sparse. Sparse matrix is one which consists of "high proportion of zero entities. A non zero threshold is select and all the pixels with intensity less than the threshold are reset to zero. As sparse matrices can be compressed heavily using Run-Length Encoding, the three sparse DC matrices are compressed using Run-Length Encoding. Thus the proposed method compresses an image at three different levels with appropriate compression algorithms to achieve higher compression ratio without compromise the image quality.

\section{a) Proposed Algorithm}

Stage1: Read the input image

Stage2: Apply Haar Wavelet Transform

Stage3: Apply Vector Quantization to Approximation Co-efficient Matrices (AC)

Stage4: Apply Thresholding to three Detail Co-efficient matrices (DC)

Stage5: Compress the Detail Co-efficient Matrices using Run-Length Coding

The process of encoding and decoding of the proposed algorithm is represented in Fig.4. and Fig.5. 
DOI: https://dx.doi.org/10.26808/rs.ca.i8v1.25

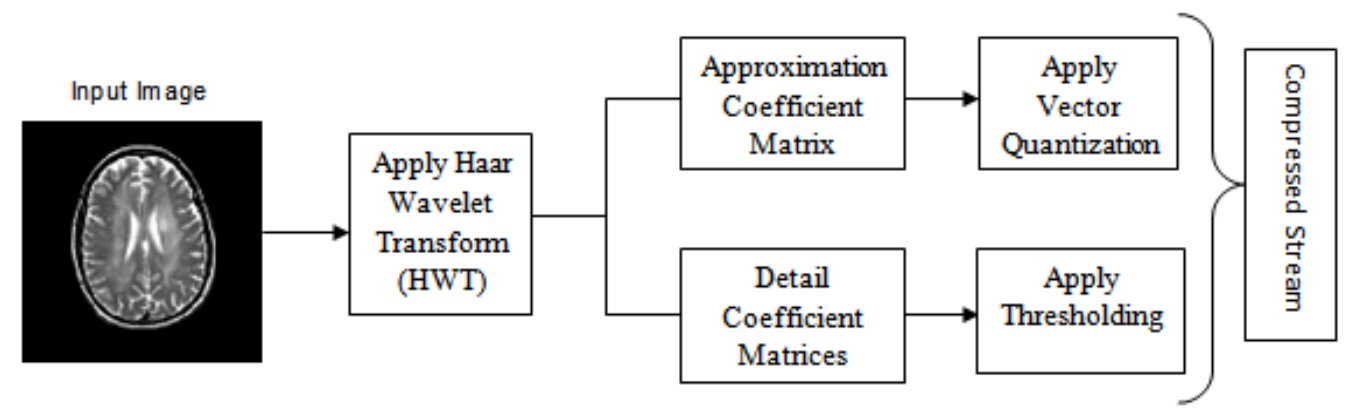

Fig.4. Encoding Process of the Proposed Method

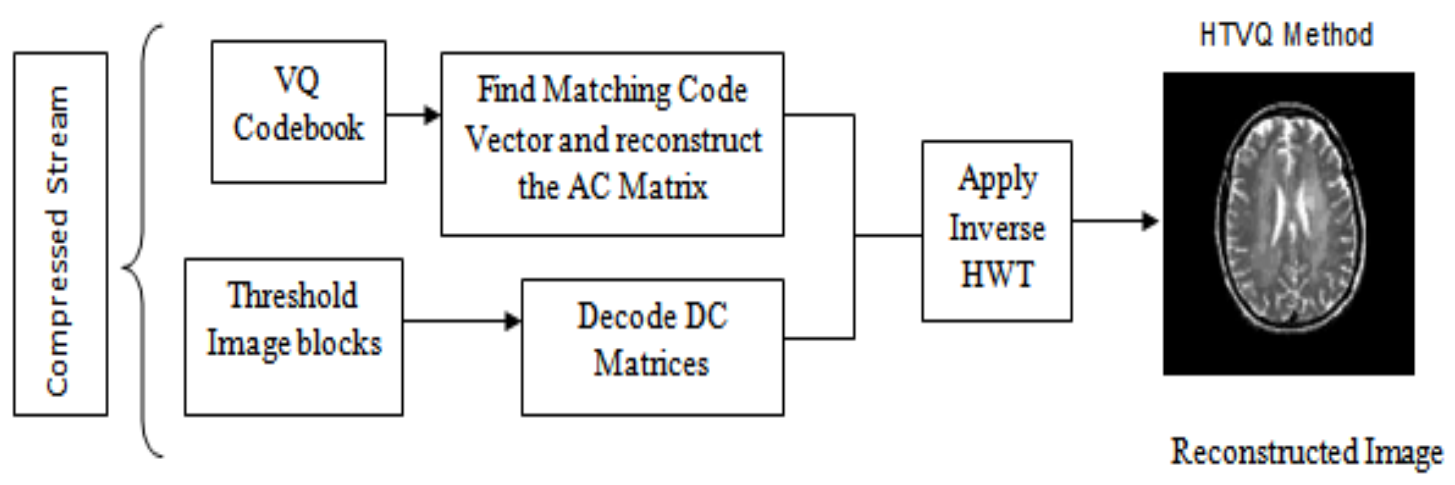

Fig 5. Decoding Process of the Proposed Method

\section{RESULTS AND DISCUSSION}

Experimental results of the proposed method is tested using five different medical test images. Evaluation process is conducted using some standard compression metrics such as Peak Signal to Noise Ratio(PSNR), Structural Similarity Index(SSIM), Compression Ratio(CR), Bit Rate (BR) and Computation Time(CT) which has proved the prominence of the proposed method.

Peak Signal Noise ratio (PSNR)- The quality of the proposed method is evaluated using PSNR value. Normally, it is measured in decibels using Eq.(3).

$$
P S N R=20 \log _{10} \frac{255}{\sqrt{M S E}}
$$

Structural Similarity (SSIM) - Similarity is measured between original and compressed image using Eq. (4)

$$
Q=\frac{4 \sigma_{\mathrm{xy}} \mu_{\mathrm{x}} \mu_{\mathrm{y}}}{\left(\sigma_{\mathrm{x}}{ }^{2} \sigma_{\mathrm{y}}{ }^{2}\right)\left(\mu_{\mathrm{x}}{ }^{2} \mu_{\mathrm{y}}{ }^{2}\right)}
$$

Compression Ratio(CR) - Compression efficiency of the proposed method is measured using Eq.(5).

$$
C R=\frac{\text { Original Image size }}{\text { Compressed Image Size }}
$$

Bit- Rate (BR) - Pixel representation of the Bit rate is calculated using Eq.(6).

$$
B p p=\frac{\text { Original Image Size in Pixels }}{\text { Total number of bits in Compressed image }}
$$

The efficiency of the proposed method is witnessed by its computation time and it must be a least value.The performance of the proposed method presented in Table 1. 
DOI: https://dx.doi.org/10.26808/rs.ca.i8v1.25 International Journal of Computer Application (2250-1797) Issue 8 Volume 1, January- February 2018

Table 1. Performance of the Proposed Method (HTVQ)

\begin{tabular}{|c|c|c|c|c|c|}
\hline \multirow{2}{*}{ Image } & \multicolumn{2}{|c|}{ Quality Metrics } & \multicolumn{2}{c|}{ Compression Performance } & \multirow{2}{*}{ CT } \\
\cline { 2 - 5 } & PSNR & SSIM & CR & BR & \\
\hline MRI_Brain & 37.343 & 0.97 & 12.63 & 0.63 & 0.92 \\
\hline MRI_Knee & 37.239 & 0.94 & 13.18 & 0.61 & 5.05 \\
\hline MRI_Spine & 44.593 & 1.00 & 12.82 & 0.62 & 6.84 \\
\hline Mammogram & 38.251 & 0.94 & 12.77 & 0.63 & 0.41 \\
\hline X-ray Image & 40.753 & 1.00 & 13.15 & 0.61 & 0.82 \\
\hline
\end{tabular}

Table 1 demonstrates the better performance of the proposed method based on the quality metrics. At the same time, compression performance also witnessed the enhanced performance of the proposed method. As the desired range of PSNR for medical image diagnosis is (30-40)db, this method is a great choice for medical image compression. The comparative analysis of the proposed method is tabulated in Table 2.

Table 2. Comparison between Existing [14] and Proposed Method

\begin{tabular}{|c|c|c|c|c|}
\hline Methods & File size & CR & MSE & PSNR \\
\hline SBC & 507540 & 0.9982 & 4.757 & 41.356 \\
\hline SVD & 507544 & 0.9966 & 26.562 & 33.888 \\
\hline DCT & 507544 & 0.9966 & 33.346 & 32.900 \\
\hline SPHIT & 507540 & 0.9982 & 6.110 & 40.269 \\
\hline NN & 507544 & 0.9966 & 12.482 & 37.167 \\
\hline VQ & 507408 & 0.9984 & 45.710 & 31.530 \\
\hline BTC & 507452 & 0.9983 & 11.961 & 37.353 \\
\hline Proposed Method (HTVQ) & $\mathbf{5 2 4 2 8 8}$ & $\mathbf{1 2 . 6 3}$ & $\mathbf{1 2 . 0 3 2}$ & $\mathbf{3 7 . 3 2 7}$ \\
\hline
\end{tabular}

The comparative analysis between the existing and proposed method is presented in Table 2. Table 2 examined the superior performance of the proposed method through the higher compression ratio. The performance of the comparative analysis is graphically represented in Fig.6. and Fig.7.

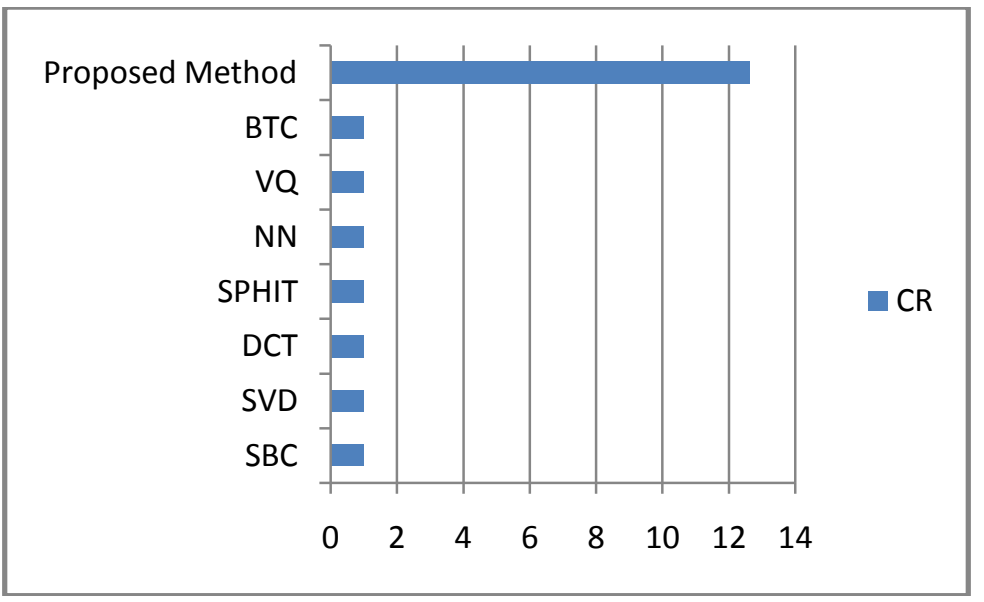

Fig 6. Comparative Analysis of Compression Ratio 
DOI: https://dx.doi.org/10.26808/rs.ca.i8v1.25 International Journal of Computer Application (2250-1797) Issue 8 Volume 1, January- February 2018

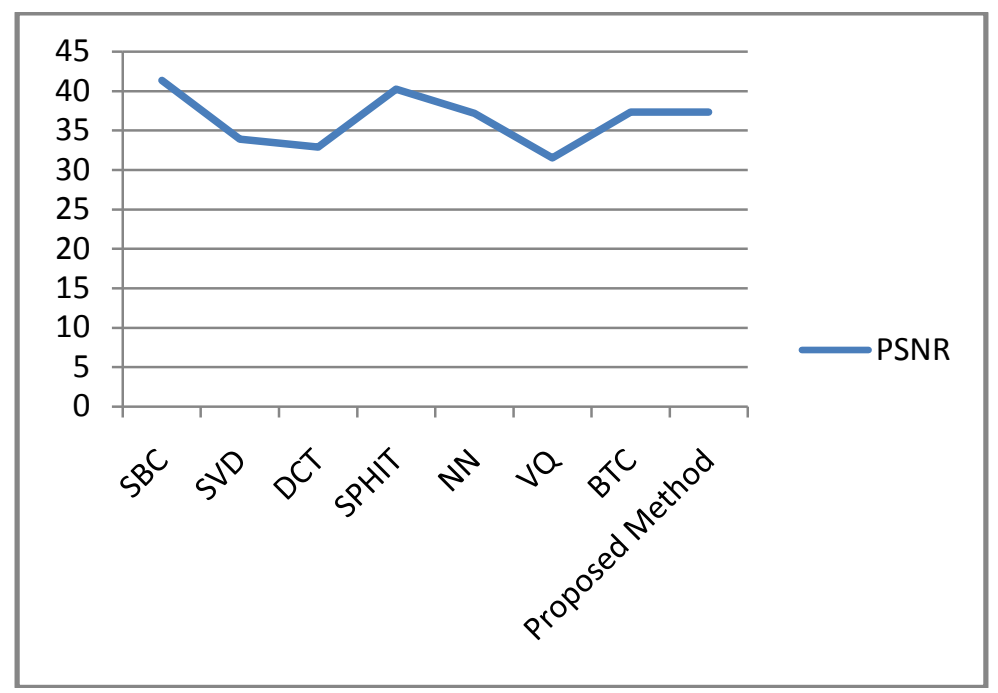

Fig 7. Comparative Analysis of PSNR Value

The visualization of the proposed method is presented in (Fig.8 - Fig 12) for five test medical images such as MRI_Brain, MRI_Knee, MRI_Spine, Mammogram and X-ray images. The achievement of the proposed method has been proved by the quality of images.

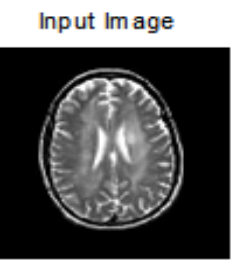

(a)

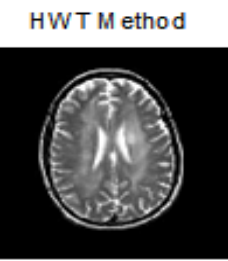

(b)

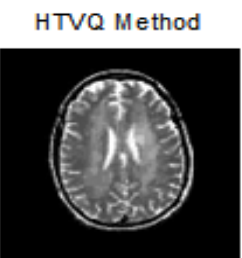

(c)

Fig.8. a) Input Image of MRI_Brain, Reconstructed Image using b) HWT Method c) Proposed Method

Input Image

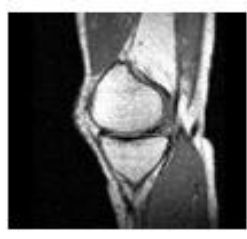

(a)
HWT M ethod

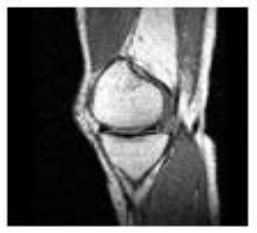

(b)

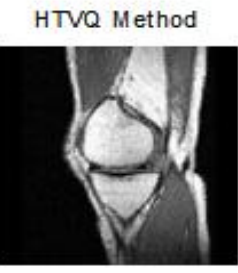

(c)

Fig.9. a) Input Image of MRI_Knee, Reconstructed Image using b) HWT Method c) Proposed Method 
DOI: https://dx.doi.org/10.26808/rs.ca.i8v1.25

International Journal of Computer Application (2250-1797)

Issue 8 Volume 1, January- February 2018

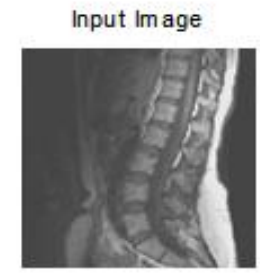

(a)

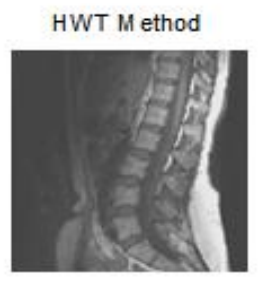

(b)

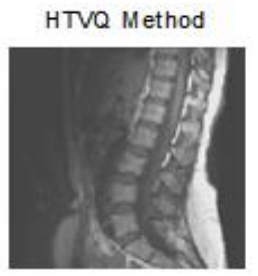

(c)

Fig.10. a) Input Image of MRI_Spine, Reconstructed Image using b) HWT Method c) Proposed Method

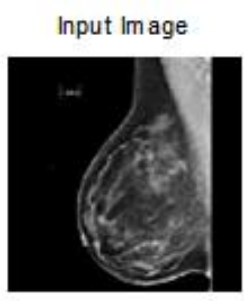

(a)

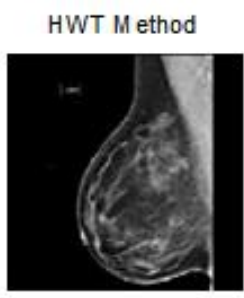

(b)

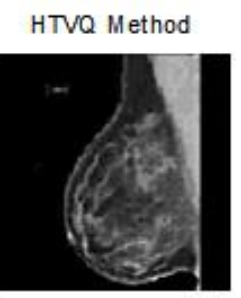

(c)

Fig.11. a) Input Image of Mammogram, Reconstructed Image using b) HWT Method c) Proposed Method

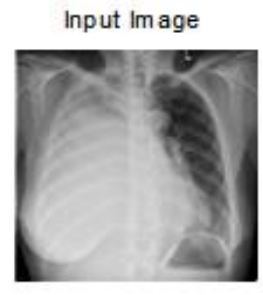

(a)

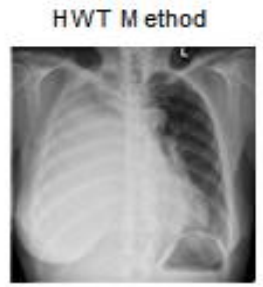

(b)

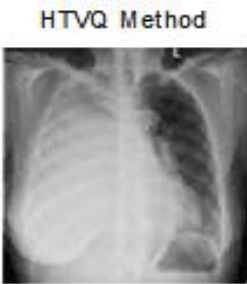

(c)

Fig.12. a) Input Image of X-ray, Reconstructed Image using b) HWT Method c) Proposed Method

\section{CONCLUSION}

In this paper, a novel method has been examined by combining HWT and VQ to enhance the compression ratio without compromising the image quality. The proposed work achieves higher PSNR and Compression ratio with acceptable range for medical diagnosis. Experiments were done for different test medical images and the performance is analyzed based on standard compression metrics. This method could be a ideal choice for transmitting the medical images. 
DOI: https://dx.doi.org/10.26808/rs.ca.i8v1.25

International Journal of Computer Application (2250-1797)

Issue 8 Volume 1, January- February 2018

\section{ACKNOWLEDGMENT}

I wish to dedicate my sincere and heartfelt thank to my research supervisor Dr.M.Mary Shanthi Rani Asst. Professor, GRI-DU who has given me a support to all my endeavour. I extend my gratitude to UGC-RGNF for providing fund for my research work.

\section{REFERENCE}

[1] K.Somasundaram and M. Mary Shanthi Rani, Novel K-means algorithm for compressing images, International Journal of Computer Applications, 18(8), pp. 9-13, 2011.

[2] K.Somasundaram and M.Mary Shanthi Rani, Eigen Value based K-means Clustering for Image Compression, International Journal of Applied Information Systems (IJAIS), Foundation of Computer Science (FCS), New York, USA, 3(7), 2012.

[3] M.Mary Shanthi Rani. Adaptive Classified Pattern Matching Vector Quantization Approach for compressing images, The 2009 International Conference on Image Processing, Computer Vision \& Pattern Recognition Proceedings, Las Vegas, USA., pp.532-538, 2009.

[4] M. Mary Shanthi Rani. Mode Based K-Means Algorithm with Residual Vector Quantization for Compressing Images , International Conference on "Control, Computation and Information Systems” (Springer-Verlag CCIS 140), pp.105-112, 2011.

[5] M.Mary Shanthi Rani, Residual Vector Quantization Based Iris Image Compression, International Journal of Computational Intelligence Studies, Inderscience Publishers, 3(4), pp.329-334,2014.

[6] M. Mary Shanthi Rani, P. Chitra, Novel Hybrid Method of Haar-Wavelet and Residual Vector Quantization for Compressing Medical Images, 2016 IEEE Conference on Advances in Computer Applications(ICACA) ,1,pp.321-326, 2016.

[7] Gopal Lakhani, Modifying JPEG Binary Arithmetic Codec for Exploiting Inter/Intra-Block and DCT Coefficient Sign Redundancies, IEEE TRANSACTIONS ON IMAGE PROCESSING, vol. 22, no. 4, pp. 13261339, April 2013.

[8] . Li, Z.-S. Wang, Compression quality prediction model for JPEG2000, IEEE Trans. Image Process, vol. 19, no. 2, pp. 384-398, Feb. 2010.

[9] G. K. Kharate, V. H. Patil, Color Image Compression Based On Wavelet Packet Best Tree, IJCSI International Journal of Computer Science Issues, vol. 7, no. 2, pp. 31-35, March 2010.

[10] Deng Wang, Duoqian Miao, Chen Xie, Best basis-based wavelet packet entropy feature extraction and hierarchical EEG classification for epileptic detection in Expert Systems with Applications, Elsevier Ltd, vol. 38, pp. 14314-14320, 2011. 
DOI: https://dx.doi.org/10.26808/rs.ca.i8v1.25

[11] M.A. Pasnur, P. S. Malge, Image Retrieval Using Modified Haar Wavelet Transform and K Means Clustering, International Journal of Emerging Technology and Advanced Engineering, vol. 3, no. 3, pp. 89-93, March 2013.

[12] Mukesh Mittal and Ruchika Lamba, Image Compression Using Vector Quantization Algorithms: A Review, International Journal of Advanced Research in Computer Science and Software Engineering Volume 3, Issue 6,pp.354-358, June 2013.

[13] S.Vimala, Codebook Enhacement For Vector Quantization Using Minmax Values for Image Compression, Ictact Journal on Image and Video Processing, Volume.04, Issue 02,November 2013.

[14] M.Mary Shanthi Rani, P.Chitra and R.Vijayalakshmi, Image Compression based On Vector Quantization Using Novel Genetic Algorithm For Compressing Medical Images, International Journal of Computer Engineering and Applications, Volume XII, Issue I, pp.104-114, 2018.

[15] M.Mary Shanthi Rani, P.Chitra and K.Mahalakshmi, A Novel Approach of Vector Quantization using Modified Particle Swarm Optimization Algorithm for Generating Efficient Codebook, International Journal of Advanced Research in Computer Science, Vol.8., No.9, 2017.

[16] J. Papitha, G. Merlin Nancy and D. Nedumaran, "Compression Techniques on MR Image - A Comparative Study", International conference on Communication and Signal Processing, April 3-5, 2013. 